\title{
COMPROMISO ORGANIZACIONAL Y SATISFACCIÓN LABORAL EN UNA MUESTRA DE TRABAJADORES DE LOS JUZGADOS DE GRANADA
}

\section{WORK ENGAGEMENT AND JOB SATISFACTION IN A SAMPLE OF WORKERS OF THE COURTS OF GRANADA}

Marta Carmen Zurita Alcaraz, $M^{a}$ Teresa Ramírez del Castillo, Juan Manuel Quesada Soto, María Elia Quesada Ronco, Bernardo Jesús Ruiz Camacho y Juan Miguel Manzano Martínez. Universidad de Granada

Artículo dirigido por: Inmaculada Valor Segura. Departamento de Psicología Social. Universidad de Granada

Fecha de recepción: 19 de marzo de 2014

Fecha de revisión: 19 de marzo de 2014.

Fecha de aceptación: 11 de abril de 2014.

\section{RESUMEN}

El objetivo de la presente investigación ha sido analizar la relación entre las variables compromiso organizacional y satisfacción laboral, esperando, como hipótesis de partida, una correlación positiva entre ambas variables, tal y como han venido demostrando estudios previos. La novedad del presente artículo reside en la muestra utilizada, 80 trabajadores de los Juzgados de Granada, con lo que se abre camino a un análisis psicológico de la situación actual de dicha organización nunca antes analizado. El diseño del estudio ha sido de tipo transeccional correlacional. Cumpliendo las expectativas, tras realizar los análisis correlacionales, se obtuvo una alta correlación positiva entre compromiso organizacional y satisfacción laboral. Además, se comprobó que no había diferencias significativas entre hombres y mujeres en cuanto a ambas variables estudiadas.

Palabras clave: satisfacción laboral, compromiso organizacional, correlación positiva, estudio transeccional.

\begin{abstract}
The objective of this research has been to analyze the relationship between work engagement and job satisfaction, hoping as hypothesis a positive correlation between both variables, as we found in previous literature. The novelty of this article has been the sample used, 80 workers of the Courts of Granada, giving a psychological analysis of the current situation of the organization never analyzed before. The study design was a correlational transectional study. Meeting expectations, after a correlational analysis we obtained a high positive correlation between work engagement and job satisfaction. Furthermore, we found that there was no significant difference between men and women in both variables.
\end{abstract}

Keywords: job satisfaction, work engagement, positive correlation, correlational transeccional study. 


\section{INTRODUCCIÓN}

En el ámbito laboral son muchas las variables psicológicas que afectan al trabajador y que tienen a su vez gran impacto en el funcionamiento de la organización. Entre estas variables, encontramos las de compromiso organizacional y satisfacción laboral, su importancia se debe a que son unos de los elementos fundamentales con los que se puede predecir la eficacia de la organización. Las organizaciones deben tener en cuenta que trabajadores satisfechos laboralmente serán trabajadores comprometidos y esto ayudará a aumentar o estimular la productividad de la organización y el funcionamiento eficaz de ellos mismos (Herzberg, 1959).

En primer lugar, podríamos definir la satisfacción laboral aludiendo a que es un conjunto de actitudes hacia el trabajo, lo que desarrolla un comportamiento y una disposición psicológica favorable o desfavorable. (Schultz, 1991) Por lo tanto, la satisfacción laboral puede ser descrita como una disposición psicológica del sujeto hacia su empleo y esto supone un grupo de actitudes hacia distintos elementos del mismo, resultado de diversas percepciones y expectativas que los empleados poseen hacia éste (Shultz, 1985), por lo que hablar de satisfacción laboral implica diferentes variables del contexto laboral (Cruz, 1999).

En la literatura sobre satisfacción laboral destaca la Teoría de los dos factores de Herzberg (1959), la cual defiende que las personas se encuentran influenciadas por dos factores: factores higiénicos o extrínsecos, cuya presencia reduce la insatisfacción, algunos aspectos de esta categoría son: el ambiente físico, el sueldo, la seguridad laboral, la supervisión o la política de la compañía. Por otro lado se encuentran los factores motivacionales o intrínsecos, y son: los logros, el reconocimiento, responsabilidad, interés en el trabajo y promoción, estos factores tendrán efecto en la presencia de la satisfacción en el individuo.

En segundo lugar, en cuanto al compromiso organizacional, éste se caracteriza por una fuerte creencia y aceptación de las metas y normas de la organización, el deseo de realizar esfuerzos significativos a favor de la organización y un fuerte deseo de seguir siendo parte de éstas (Mathieu y Zajaz, 1990). Es decir, es un estado psicológico que caracteriza la relación entre una persona y una organización, la cual presenta consecuencias respecto a la decisión para continuar en la organización o dejarla (Meyer y Allen, 1991).

Con respecto a la relación entre satisfacción laboral y compromiso organizacional, se ha encontrado gran cantidad de investigación que relaciona ambas variables; así por ejemplo, se ha estudiado la correlación entre dichas variables en una muestra del cuerpo de bomberos (Atencio, y Ortega, 2009); satisfacción y compromiso en la cultura de grupos (Topa, Lisbona, Palací y Morales, 2003); un meta-análisis de correlaciones encontradas entre satisfacción laboral y compromiso organizativo en diferentes contextos (García, y Brás, 2007); también han encontrado que la satisfacción laboral es la variable que en mayor medida pronostica el compromiso de los empleados (Agulló, Boada, González, Mañas, Salvador. 2007). En todas estas investigaciones se ha hallado una correlación positiva entre las variables de estudio.

Por otro lado, se han encontrado igualmente un gran número de artículos que relacionan dichas variables con otras que también forman parte del ámbito laboral, como pueden ser el clima laboral, el tipo de liderazgo o el síndrome de estar quemado en el trabajo 
(burnout); de hecho para esta última variable los resultados muestran la existencia de correlación significativa entre satisfacción laboral y los tres tipos de dimensiones de burnout (agotamiento emocional, despersonalización y realización personal). (Camacho, García, León, Mateos, Peña, 2013). También, destacamos los resultados encontrados en investigaciones previas con respecto a la relación entre satisfacción y compromiso con el liderazgo, se muestra una relación positiva entre el empleo de las conductas de liderazgo, satisfacción en el trabajo y el compromiso organizacional de los empleados (Villalba, 2001).

Vista la investigación previa existente, el objetivo del presente artículo ha sido analizar la correlación entre satisfacción laboral y compromiso organizacional, esperando y corroborando una correlación positiva tal y como muestran los resultados de estudios anteriores. Como novedad, esta investigación aporta el hecho de haber analizado dichas variables en una muestra de trabajadores de los Juzgados de Granada. Se trata de una muestra homogénea, los empleados pertenecen a una misma organización con similares condiciones de trabajo: mismos horarios, funciones y actividades muy semejantes (aunque con ligeras variaciones según el puesto desempeñado por cada uno de los miembros de la muestra); con esto se espera obtener unos resultados claros en el análisis de las variables de estudio evitando así la presencia de posibles variables extrañas.

Tradicionalmente la satisfacción del ciudadano con la administración de justicia ha dejado mucho que desear, debido fundamentalmente al gran retraso en la resolución de los procedimientos. Si bien es cierto que esta tendencia ha ido variando en los últimos años como consecuencia de numerosas reformas legales, especialmente en materia penal y civil (prueba de ello es que actualmente un delito no grave puede resolverse en un período de no más de quince días), no obstante en los asuntos de especial dificultad sigue habiendo retraso por problemas que no siempre son imputables a los órganos jurisdiccionales, sobre todo debidos a la falta de medios y excesiva acumulacion de trabajo. Este estudio permite acercarse desde una perspectiva psicológica, antes no abordada, a la situación de los trabajadores de los Juzgados de Granada; en concreto hace posible observar en los empleados de esta organización dos variables psicológicas, la satisfacción laboral y el compromiso con la organización. Aunque nuestro objetivo sea analizar la correlación entre estas dos variables, se aportan datos de interés que abren camino a que estudios futuros puedan establecer si el nivel satisfacción y compromiso observado es causado por la mencionada situación actual de esta organización.

No es la primera vez que en el sector público se encuentra una situación laboral mejorable, y no solo en España sino también en otros países como por ejemplo Chile, donde una serie de condiciones como la falta de infraestructuras, el aumento de población penal o la inadecuada penalización de los delitos afectan a la organización del sistema penitenciario (Bravo y Jiménez, 2007); con este ejemplo queremos hacer alusión a que sería conveniente estudiar que las variables psicológicas observadas en los trabajadores de una organización no están determinadas exclusivamente por los factores intrínsecos sino que son muchas veces los factores externos los que influyen sobre los internos.

Por tanto, como ya hemos mencionado, partimos de la hipótesis en este estudio de obtener una correlación positiva entre las variables satisfacción laboral y compromiso organizacional en dicho contexto. Paralelamente a este objetivo principal también se han propuesto otros. Así, otro objetivo del que partimos será analizar si existen diferencias entre sexos en cuanto al nivel de satisfacción laboral y compromiso organizacional; respecto a esta variable género, en otras investigaciones previas se ha encontrado que 
hay diferencias significativas en la satisfacción manifestada entre varones y mujeres de la muestra, detectando niveles sensiblemente inferiores en los primeros (Artacho, Fuentes y Sánchez, 2007). Por otra parte también se tratará de averiguar si existen diferencias en cuanto a la satisfacción laboral y compromiso organizacional entre distintos grupos de miembros de la organización según sus respectivos años de antigüedad en ésta y entre la edad de los participantes de la muestra.

\section{MÉTODO}

\section{- Participantes:}

La muestra con la que se ha realizado esta investigación está compuesta por 80 funcionarios públicos de los Juzgados de Granada, pertenecientes a los cuerpos de gestión, tramitación y auxilio judicial; cuyas funciones y responsabilidades varían en función de su cargo profesional. Las edades de los participantes están comprendidas entre los 33 y los 58 años $(M=48 D T=4,6)$. En cuanto al sexo, el $31,3 \%$ de la muestra estaba compuesta por hombres, frente al $68,8 \%$ de mujeres. Todos los participantes de la investigación son de nacionalidad española y residentes en Granada.

\section{- Diseño:}

Se ha llevado a cabo una investigación no experimental de tipo transeccional correlacional, pues el objetivo en este estudio es el de describir la relación entre dos variables de forma correlacionada y en un momento temporal determinado.

\section{- Instrumentos:}

Para llevar a cabo la presente investigación se utilizaron dos cuestionarios de tipo Likert. Por una parte, el cuestionario de compromiso organizacional de Allen y Mayer (1997); Modificación Cedeño y Pirela (2002), el cual consta de 18 afirmaciones o juicios positivos y negativos como "Esta organización merece mi lealtad" o "No me siento como parte de la familia en mi organización", que definen las tres dimensiones que constituyen el compromiso organizacional: componente afectivo, normativo y de continuidad, ante las cuales el sujeto debe puntuar del 1 al 7 el grado de acuerdo o desacuerdo que mejor describe su actitud ante tales afirmaciones, el valor 1 indica totalmente en desacuerdo y el 7 totalmente de acuerdo. Con respecto al coeficiente de fiabilidad de este cuestionario, en el presente estudio, medido a través del alfa de Cronbach resultó ser de 0, 68 .

Por otra parte se utilizó el cuestionario de Satisfacción S20/23 (Meliá y Peiró, 1989).

Los ítems se responden con una escala de 7 puntos que valora el grado de satisfacción que el trabajador tiene con diferentes aspectos del trabajo como: satisfacción con la supervisión (6 ítems: relaciones con supervisor y con la supervisión recibida), satisfacción con el ambiente físico de trabajo (5 ítems: limpieza y espacio disponible), satisfacción con las prestaciones (5 ítems: salario, promoción y formación), satisfacción intrínseca (4 ítems: hacer lo que le gusta y objetivos que debe alcanzar) y satisfacción con la participación (3 ítems: capacidad para decidir y participación en decisiones del departamento). La escala permite obtener una puntuación global en satisfacción laboral resultado del promedio de las puntuaciones de los ítems que la forman. Altas puntuaciones indican mayor satisfacción laboral. En relación a la fiabilidad de este cuestionario en esta investigación, se realizó el alfa de Cronbach con un resultado obtenido de 0,91. 


\section{- Procedimiento:}

Para acceder a la muestra en los Juzgados, se informó previamente a uno de los secretarios judiciales del objetivo y las características de la investigación, tras recibir su conformidad, se procedió a pedir la colaboración voluntaria y anónima a los trabajadores del Juzgado de Granada. Una vez que éstos accedieron, se les administró los cuestionarios, informándoles que el objetivo era realizar un trabajo de investigación para la asignatura "psicología del trabajo" de la Facultad de psicología de Granada, además se les explicaron las instrucciones de cada uno de los cuestionarios. Los participantes rellenaron los instrumentos proporcionados en sus correspondientes puestos de trabajo, con una buena iluminación, temperatura agradable y en un horario donde aún no atendían al público, evitando de esta manera posibles distracciones. Al día siguiente, procedimos a la recogida de los cuestionarios agradeciendo a todos los participantes su colaboración.

\section{RESULTADOS}

El análisis de datos en este apartado se realizó con el paquete estadístico SPSS 22.0 para Windows. El objetivo último fue el de mostrar, tras haber cuantificado las variables y haber utilizado el procedimiento estadístico adecuado, si existía una relación cuantitativamente significativa entre los datos obtenidos en compromiso organizacional y satisfacción laboral, a nivel general. Pero, antes de nada, se van a exponer los siguientes estadísticos aplicados a las distintas variables que fueron analizadas en la muestra de este estudio en particular:

\section{Mínimo Máximo Media D. Típica}

$\begin{array}{lllll}\text { Edad } & 33 & 58 & 48,5375 & 4,62504\end{array}$

Antigüedad 3

32

$21,1125 \quad 5,87420$

Compromiso organizacional

2,22

6,22

4,3042

0,82790

Satisfacción laboral

2,04

5,87

3,8755

0,81110

Tabla 1. Estadísticos descriptivos.

De acuerdo con los baremos del cuestionario de compromiso organizacional, se constató que el nivel de compromiso general de la muestra de trabajadores del Juzgado de Granada era medio ( $M=4.30 ; \mathrm{DT}=.83)$.

Por su parte, en satisfacción laboral se plasmaron los resultados obtenidos a nivel global, pese a que este cuestionario se podía dividir en subescalas lo que interesa para esta investigación era la puntuación general, la cual se encontraba también en un nivel medio $(\mathrm{M}=3.87$; $\mathrm{DT}=.811)$.

Con el uso de la correlación bivariada de Pearson, se encaminó la búsqueda hacia la existencia o no de correlación positiva entre compromiso organizacional y satisfacción 
laboral. Lo que los resultados desvelaron, tras el uso de este procedimiento estadístico, es que sí que existía correlación cuantitativamente significativa entre estas variables, y además, de forma positiva $(r=.41 ; p<.01)$. Esto quiere decir que, en general, los sujetos que puntuaron alto en compromiso organizacional, así lo hicieron en satisfacción laboral, y esto mismo ocurría si los individuos estudiados puntuaban al contrario.

En última instancia, se procedió a constatar si existían diferencias entre distinto sexo tanto en el compromiso con la organización como en satisfacción laboral. Para ello, se utilizó una $\mathrm{t}$ de student para muestras independientes, que reflejó que no había diferencias significativas entre hombres y mujeres en relación al compromiso con la organización $t(.297)=-1.051 ; p<.05$, así como mostró la ausencia de diferencias significativas en la satisfacción laboral entre ambos sexos $t(.416)=-.819 ; p<.05$. A continuación, se plasman estas similitudes encontradas entre hombres y mujeres:

\begin{tabular}{lcccc}
\hline & sexo & N & media & Desviación estándar \\
Compromiso laboral & Hombre & 25 & 4,1600 & 0,78146 \\
& Mujer & 55 & 4,3697 & 0,84695 \\
Satisfacción laboral & Hombre & 25 & 3,7652 & 0,87354 \\
& Mujer & 55 & 3,9257 & 0,78429 \\
\hline
\end{tabular}

Tabla 2. Diferencias entre sexo.

\section{DISCUSIÓN}

A lo largo de la investigación hemos intentado averiguar la correlación existente entre satisfacción laboral y compromiso organizacional; los resultados obtenidos permiten concluir la existencia de una correlación significativa entre las dos variables $(r=.41)$, es por tanto que se corrobora la hipótesis de partida.

De acuerdo con los resultados, se obtiene que el nivel de compromiso general de la muestra de trabajadores con el Juzgado de Granada es medio. Por tanto, dada la correlación positiva observada, la satisfacción laboral también se encuentra en un nivel medio. Encontramos además en ambas variables una desviación típica baja, indicando homogeneidad de respuestas de la muestra con respecto a la media.

Dada la alta correlación encontrada, esto nos permitiría plantear la posibilidad de que al mejorar el compromiso con la organización o bien la satisfacción laboral de los trabajadores del Juzgado de Granada, se beneficiaría de ello la otra variable psicológica analizada en esta investigación. De este modo, también existe la necesidad de tomar precauciones para evitar la disminución de alguna de estas dos variables por parte de los trabajadores, ya que esto podría tener repercusiones negativas en un distintivo número de variables psicológicas diferentes, por ejemplo pudiendo afectar al rendimiento laboral. 
Estos datos aportan información de importancia para el contexto organizacional de los Juzgados de Granada, ya que si esta organización conoce el nivel general de satisfacción laboral y compromiso organizacional de sus trabajadores (medio según nuestros resultados) podrá tomar medidas para estimular y tratar de aumentar el nivel de dichas variables, lo que se traduciría en un beneficio para la organización al aumentar a su vez otras, como la variable rendimiento, que como ya hemos visto, también correlaciona con las estudiadas.

Por otro lado, también se ha analizado si el nivel satisfacción laboral y el compromiso organizacional, mostrados por la muestra, variaba en función del sexo $(31,3 \%$ hombres, $68,8 \%$ mujeres): los resultados no han mostrado diferencias significativas por lo que el género no ha influido en las variables del estudio.

En este estudio, en un primer momento se planteó la posibilidad de analizar si existían diferencias en cuanto a la satisfacción laboral y compromiso organizacional entre los miembros de la organización según sus distintos años de antigüedad en ella, por un lado, y entre la edad de los participantes de la muestra, por el otro; (datos analizados en la tabla 1 de los resultados). Posteriormente se rechazó la idea de analizar dichas diferencias ya que tanto los años de antigüedad como la edad eran muy similares entre todos los sujetos de la muestra, no pudiendo establecer grupos de edad ni antigüedad diferentes. Por otra parte, a pesar de no haber podido establecer si este tipo de variables puede influir en la satisfacción laboral y compromiso organizacional de los trabajadores de nuestra muestra dadas sus condiciones, en el caso de que las variables edad y antigüedad influyeran realmente en nuestras variables de estudio, el hecho de ser nuestra muestra tan homogénea en este aspecto, habría permitido que dichas variables edad y antigüedad no influyan como variables extrañas haciendo menos visible la similitud entre los niveles de satisfacción y compromiso observados entre todos los participantes de la muestra.

En otros estudios hemos encontrado la influencia de la variable edad; (Ramírez, 2002) concluye que a medida que aumenta la edad los empleados están más motivados para implicarse en los trabajos que desarrollan. Además la relación entre edad y satisfacción es positiva a partir de intervalos de 31-40 años, alcanzando el máximo de satisfacción en el intervalo previo a la jubilación (Marín, 1981).

Con respecto a las limitaciones del estudio y, en concreto, las diversas variables que han sido imposibles de controlar, a continuación se van a exponer las que consideramos más relevantes:

Debido a que esta investigación es un estudio correlacional, la inclusión de este proceder implica dificultades a la hora de determinar la existencia de causalidad en la relación de estas variables, así como también dificulta determinar la dirección de la relación entre las variables y el control de terceras variables explicativas de la supuesta relación.

Hay que poner de manifiesto la dificultad que constataron la mayoría de los trabajadores de la muestra para poder completar el cuestionario en buenas condiciones, debido a que durante la administración de las pruebas éstos se encontraban en su puesto de trabajo y no tenían demasiado tiempo disponible, con lo que ha podido afectar a la atención, concentración y tiempo dedicados a la óptima elaboración de los cuestionarios. Por otro lado, pese a que se intentó seleccionar instrumentos que evaluaran las variables 
requeridas de la forma más breve y sencilla posible, algunos trabajadores revelaron que la extensión de los cuestionarios les parecía excesiva y que en algunos casos algunos ítems les resultaban algo ambiguos, tal vez este comentario se debiera a su falta de tiempo disponible. Además, se ha considerado la posibilidad de que ciertos trabajadores mantuvieran una falta de interés real por colaborar en la investigación, ante la proximidad de las vacaciones de Navidad, cuando los niveles de estrés y ocupación laboral son más elevados, según mantenía una amplia mayoría de los encuestados. Todo esto hace referencia a problemas ocasionados con la aplicación del instrumento, que sin lugar a duda son de gran importancia, pero que no ha sido posible su control.

Lo más adecuado hubiera sido por tanto controlar la forma en la que se aplican dichos instrumentos facilitando a los trabajadores la realización de los mismos haciendo, por ejemplo, que los puedan completar en un límite mayor de tiempo y en otro contexto que no sea el laboral.

Como ya mencionamos, este estudio abre paso a posibles líneas de investigación futuras sobre este contexto organizacional; en concreto proponemos que se estudie si la situación actual de los Juzgados antes referida (falta de medios y excesiva acumulacion de trabajo), es la causante de los resultados aportados por este estudio en los que encontramos que los niveles de satisfacción y compromiso organizacional son medios.

En cuanto a la influencia de las variables edad y antigüedad sobre las de satisfacción y compromiso también sería interesante desarrollar estudios posteriores en los que en la muestra seleccionada se pueda establecer distintos grupos de edad y antigüedad.

Otro tipo de líneas de investigación futuras que proponemos podría ser el análisis del comportamiento de ambas variables de estudio a lo largo del tiempo, con lo que con ello también se podría observar si el cambio de condiciones organizacionales a lo largo del tiempo, y en concreto la consideración de qué cambios particulares, generan o no un aumento o decremento en el nivel de las variables de estudio. Por otra parte también se podría analizar la influencia de otro tipo de variables junto con las ya descritas.

\section{REFERENCIAS}

Agulló, E., Boada, J., González, E., Mañas, M.A., y Salvador, C. (2007). La satisfacción y el bienestar psicológico como antecedentes del compromiso organizacional. Psicothema, 19 (3), 395-400.

Artacho, C., Fuentes, F. y Sánchez, S. ( 2007). La perspectiva de género en el análisis de la

satisfacción laboral: una aplicación empírica mediante modelos logit y probit. Cuadernos de gestión, 7 (2), 55-67.

Atencio, S; y Ortega, H. (2009). Satisfacción laboral y compromiso organizacional en el cuerpo de bomberos. Trabajo especial de grado, Universidad Rafael Urdaneta, Maracaibo, Venezuela.

Bravo, C., Jiménez, A. (2007) Bienestar psicológico, apoyo organizacional percibido y satisfacción laboral en funcionarios penitenciarios de Chile. Scielo, 13nov.-feb. 2011 
Cruz, S. C. (1999). La Satisfacción en el Trabajo y su relación con la Motivación. (Tesis de Maestría) México: Universidad Nacional Autónoma de México, Facultad de Contaduría y Administración

García, D., Peña, M.P., León, E., Camacho, B., y Mateos, A., (2013) Relacion entre las dimensiones del síndrome de burnout y los factores de satisfacción laboral. Reidocrea. 2, 33-40.

García, J, . y Brás, J.M. (2008). Satisfacción profesional y compromiso organizativo:

un meta-análisis. Revista Europea de Dirección y Economía de la Empresa, 17(3), 61-78.

Herzberg, F. (1959). The Motivation to Work. New York: John Wiley and Sons.

Marín, L (1981). .Una aproximación sociológica al estudio de la satisfacción en el trabajo en la provincia de Santander. Reis, 13, 99-100.

Mathieu, J. E. y Zajac, D. M. (1990). A review and meta-analysis of the antecedents, correlates and consequences of organizational commitment. Psychological Bulletin, 180, 171-194

Meliá, J.L., y Peiró, J.M. (1989). La medida de la satisfacción laboral en contextos organizacionales: El Cuestionario de Satisfacción S20/23. Psicologemas,5,59-74

Meyer, J.P. y Allen, Natalie J. (1991). A three component conceptualization of organizational commitment. Human Resource Management Review, 1, 61-98.

Ramirez, R. (2002). Condicionantes sociolaborales de los empleados públicos: motivación y satisfacción laboral en la Administración Regional de Extremadura. Tesis doctoral. Universidad de Extremadura.

Schultz, D. P. 1985. Psicología Industrial. México: Interamericana

Schultz, D. P. (1991). Psicología Industrial. México: Edit. Me Graw Hill.

Topa Cantisano, G., Lisbona, A, Palací, F. y Morales, F. (2003). Percepción de ruptura de contrato psicológico y burnout en funcionarios de instituciones penitenciarias españolas: mediación de variables personales y organizacionales. $8^{\circ}$ Congreso Nacional de Psicología Social. Torremolinos. España.

Villalba, O. (2001). Incremento de la satisfacción y del compromiso organizacional de los empleados a través del liderazgo efectivo. Academia. Revista Latinoamericana de Administración, . 5-17. 